\title{
ERRATUM, VOLUME 72
}

Fred Gross, On the equation $f^{n}+g^{n}=1$, pp. 86-88.

In Theorem 2 the expressions for $f$ and $g$ are incorrect and should be:

$$
\begin{aligned}
& f=\frac{1}{2 \varphi}\left(1+3^{-1 / 2} \varnothing^{\prime}\right), \\
& g=\frac{-1}{2 \varnothing}\left(1-3^{-1 / 2} \varnothing^{\prime}\right) .
\end{aligned}
$$

In line 7 of the proof of Theorem $2 F$ and $G$ should be interchanged. That is, it should read

$$
F=4^{1 / 3} /(f+g) \text { and } G=3^{1 / 2}(f-g) /(f+g) .
$$

